\title{
LA VARIABLE DE GÉNERO EN LA ENSEÑANZA DE IDIOMAS EN EL AULA MULTICULTURAL. EL APOYO DE LAS TIC
}

\author{
THE VARIABLE GENDER IN THE LANGUAGE TEACHING IN THE MULTICUL- \\ TURAL CLASSROOM. THE SUPPORT OF TIC
}

Elisa María Casero Osorio

UNED

\section{Resumen:}

La multiculturalidad es un hecho que está cada vez más presente en nuestros centros educativos. Países como España han acogido un gran número de inmigrantes desde la década de 1990 por lo que el sistema educativo español ha tenido que adaptarse para que este colectivo se incorpore, satisfactoriamente, a nuestras aulas. Conseguir la heterogeneidad, es decir, que las diversas culturas convivan de manera conjunta, es un objetivo en los centros educativos. Pero no debemos olvidar una lucha que ya había comenzado con anterioridad: conseguir la plena igualdad entre hombres y mujeres. Centrándose en la enseñanza de idiomas, este artículo estudia si existen, en el presente, diferencias entre sexos en la nueva aula multicultural y cómo las tecnologías de la información y comunicación junto con el uso de la taxonomía de Bloom, como herramienta para establecer objetivos de aprendizaje, pueden servir para conseguir los principios básicos de tolerancia, respeto e igualdad en centros educativos.

\section{Palabras claves:}

Multiculturalidad; igualdad; género; idiomas; TIC; Bloom

\section{Abstract:}

Multiculturalism is increasingly present in our schools and education centers nowadays. Several countries as Spain have welcomed a large number of immigrants since the 1990s, so that the Spanish educational system experienced an adaptation process to facilitate that this group is successfully incorporated into our classrooms. Achieving heterogeneity, that is, the various cultures coexisting together, is currently a main goal in schools. But we don't have to forget that this struggle already existed: to reach full equality between men and women. By means of language teaching, this article studies if there currently exist a gender bias in the new multicultural classrooms, and how information and communication technologies, in addition with Bloom's taxonomy use, act as a tool for establishing new learning objectives, and even achieve the basic principles of tolerance, respect and equality in education centers.

\section{KEY WORD:}

Multiculturalism; equality; gender; language; TIC; Bloom. 


\section{Multiculturalidad. LA LLEgada DE INMigrantes}

Según la Real Academia de la Lengua Española, el concepto "multicultural" se define como "la convivencia de diversas culturas". El término "multiculturalidad" es una cualidad de multicultural, definido como (Song, 2010) "Multiculturalism is a body of thought in political philosophy about the proper way to respond to cultural and religious diversity", es decir, es la forma de nombrar la diversidad lingüística y religiosa en un territorio.

El fenómeno de la multiculturalidad se ha expandido por casi todos los territorios del planeta. Siguiendo las teorías de Sales y García (1997) el fenómeno de la multiculturalidad nace a partir de una serie de sucesos que tuvieron lugar en las últimas décadas del siglo XX. Destacan los siguientes:

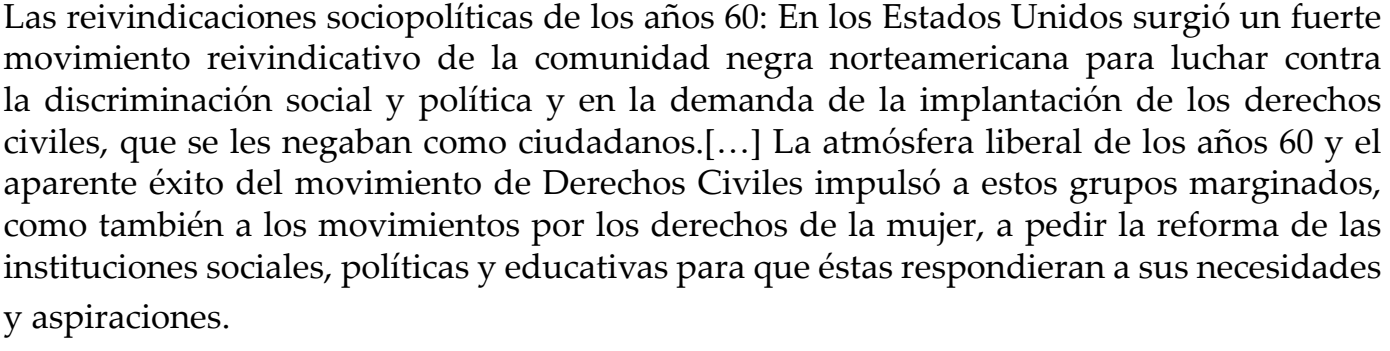

El auge del factor étnico: El concepto de etnicidad se está convirtiendo en uno de los factores de presión sociopolítica más importantes de esta segunda mitad de siglo, junto a los de clases social y género [...] Esta conciencia étnica, unida a la idea de pluralismo democrático de los Estados actuales es la que ha dado lugar a las reivindicaciones políticas anteriormente citadas, que han ido conformando la compleja realidad multicultural de nuestros días.

El fenómeno migratorio y la problemática del llamado Tercer Mundo: Desde los años 60 y 70 los países industrializados, sobre todo Alemania, Francia, los Estados Unidos, Suecia, Inglaterra, Suiza y Australia, han visto aumentar la población inmigrante de una manera acelerada, lo que conlleva la necesidad de planteamientos políticos respecto a esta población crecientemente multicultural.

La independencia mundial: La apertura de fronteras, la facilidad y rapidez en las comunicaciones y la información, y la globalización de los problemas políticos y económicos hacen cada vez más interdependientes a todos los países del mundo, lo que exige, a su vez, mayores y mejores relaciones interculturales.

Como se puede observar, no es posible decir que la multiculturalidad sea un fenómeno reciente. En países como España el mayor flujo de entrada de inmigrantes se produjo a partir de los años 90 debido a factores como el desarrollo económico a partir de esta década con un incremento en actividades como la construcción y el turismo, la suavidad del clima, la atracción por el modo de vida, la cercanía del continente africano y la similitud lingüística y cultural con Iberoamérica. Hoy en día, España cuenta con una población inmigrante que asciende a la cantidad aproximada de 4.500.000, repartidos en todo el territorio y causando una diversidad social, económica, política y cultural bastante importante. Como explican Godenau (2014) en La integración de los inmigrantes en España: una propuesta de mediación a escala regional, son las Comunidades Autónomas que componen el territorio español las encargadas de fijar las medidas necesarias en todos los ámbitos para la correcta integración de este colectivo

Las CC.AA. españolas han ido adquiriendo importantes competencias sectoriales, por ejemplo en materias como educación, sanidad y cultura, así como en políticas sociales (de empleo, vivienda, servicios sociales, etc.) Este reparto competencial hace que a las CC.AA. les corresponda tomar medidas que repercuten en la integración social y económica de los colectivos inmigrantes, así como su participación social. Es por ello que en España, en una mayoría de CC.AA. se han elaborado planes regionales de integración.

Campos como la educación, han evolucionado a gran velocidad para incorporar a este colectivo de inmigrantes a las aulas españolas conocidas, actualmente, como multiculturales. Hoy en día se aplican medidas como aulas de inversión lingüística, la creación de la figura del mediador intercultural, programas de acogida, centros de recursos específicos y formación del profesorado, entre otras muchas.

\section{IGUALDAD DE GÉNERO EN CENTROS ESPAÑOLES. EL CASO INMIGRANTE}

Según la página oficial de la UNESCO", "la cuestión de género debe ser considerada prioritaria en la planificación de la educación, desde las infraestructuras hasta el desarrollo de materiales o los procesos pedagógicos. La participación total y equitativa de las mujeres es vital para asegurar un futuro sostenible". En la escuela, no debe existir una separación de géneros, todos los alumnos y alumnas deben ser educados en igual de condiciones. Pero, ¿cuáles son los motivos por los que se debe educar en género en los centros escolares? La misma página de la $\mathrm{UNESCO}^{2}$ ofrece información para clarificar esta cuestión.

- Los roles de género son creados por la sociedad y se aprenden de una generación a otra;

1 Página oficial de la UNESCO que trata sobre temas de Educación, en este caso, la Educación para el desarrollo sostenible, la igualdad de género.

2 http://www.unesco.org/new/es/education/themes/leading-the-international-agenda/ education-for-sustainable-development/gender-equality/ 
- Los roles de género son constructos sociales y se pueden cambiar para alcanzar la igualdad y la equidad entre las mujeres y los hombres;

- Empoderar a las mujeres es una herramienta indispensable para hacer avanzar el desarrollo y reducir la pobreza;

- Las desigualdades de género socavan la capacidad de las niñas y mujeres de ejercer sus derechos:

- Asegurar la igualdad de género entre niños y niñas significa que ambos tienen las mismas oportunidades para acceder a la escuela, así como durante el transcurso de sus estudios.

En anteriores décadas, la sociedad, en este caso española -aunque ampliable a otras sociedades fuera de nuestras fronteras-, se caracterizaba por ser patriarcal, es decir, el género masculino era el que gobernaba en la casa y fuera de ella, relegando a la mujer a un segundo plano. Hasta el año 1933, las mujeres no consiguieron el sufragio activo o derecho al voto. Desde entonces, el rol de la mujer ha cambiado positivamente a gran velocidad pero, hoy en día, quedan vestigios de aquella sociedad patriarcal de hace apenas 50 años; además, la llegada de un colectivo inmigrante muy numeroso procedentes de países, algunos de los cuales, dentro de sus culturas siguen manteniendo la supremacía del hombre sobre la mujer, son motivos por los que, desde la escuela, es necesario remarcar que ya no deben existir esos roles de género que separan los papeles entre hombres y mujeres en todos los ámbitos de la vida.

En el ámbito de educación, los roles de género también han estado diferenciados hasta bien entrado el siglo XX. Como bien resume la doctora Del Amo (2009: 9) hasta pasada la segunda mitad del siglo XX, no se experimenta un avance notable en la educación de mujeres jóvenes.

El ritmo de escolarización y de alfabetización de las niñas antes del siglo XX fue lento y los contenidos, los espacios y horarios fueron diferentes a los de los varones. Desde mediados del siglo XIX la ley establece que todas las niñas deben acudir a la escuela primaria -sin embargo, el absentismo escolar entre las niñas era elevadísimo y las escuelas escasas-, una exigua minoría empieza a frecuentar las aulas de los institutos, algunas jóvenes cursan estudios musicales, de magisterio o de matrona y unas pocas se atreven a pisar las aulas universitarias. Una vez que algunas consiguieron concluir con éxito los estudios superiores, todavía fue necesario esperar más tiempo para que se aceptase que los conocimientos adquiridos se pusieran al servicio del ejercicio profesional. Si bien durante el primer tercio del siglo $\mathrm{XX}$ y, particularmente, durante la II República la educación femenina experimenta un avance notable, el franquismo supuso una inflexión hasta que la Ley de Educación de 1970 y la posterior legislación educativa de la España democrática implantan la escuela mixta y la igualdad formal de oportunidades entre los sexos.
A principios del siglo XX, la situación de las mujeres en el ámbito educativo era muy diferente a lo que conocemos hoy en día. Para aquellas que decidían estudiar y, como explica Flecha (2014:52) "hasta 1910 era necesaria la solicitud de permisos para poder acudir a las clases mixtas; cuando se lograba, las mujeres debían ocupar lugares separados en las aulas, y cuando obtenían el título los colegios profesionales impedían el ejercicio profesional en las mismas profesiones que a sus compañeros." Si bien en un principio se reconoció la necesidad de que las niñas fueran al colegio, sus materias eran muy distintas a las de sus compañeros varones; además de aprender a leer y a escribir, estas niñas debían conocer todo lo relativo a la domesticidad, mientras que los niños se dedicaban a otras actividades. Pero las continuas ampliaciones de escolaridad obligatoria (doce años en 1909 y dieciséis años en 1990) supusieron que estas niñas retrasasen su incorporación a las tareas domésticas y comenzaran a tener más curiosidad por otros oficios. Realmente no es hasta el año 1990, con la implantación de la Ley Orgánica de Ordenación General del Sistema Educativo (LOGSE), que se reconoce la discriminación por sexos y se establece la necesidad de reconsiderar la actividad educativa en base a los principios de igualdad de oportunidades. Actualmente, para el año 2014, según datos estadísticos del Ministerio de Educación, Cultura y Deporte, el porcentaje de mujeres con nivel de formación de educación secundaria, entre 25 y 34 años, es de un $71,2 \%$, frente al $38 \%$ que encontramos en mujeres con edades comprendidas entre 55 y 64 años - nacidas en los años 50, cuando la educación de la mujer giraba en torno al saber de las tareas domésticas y cuidado de los hijos-. En cuanto a la educación superior, el porcentaje de mujeres ente los 30 y 34 años se sitúa en este mismo año, en el $47,8 \%$, frente al $36,8 \%$ de hombres. Claramente, podemos ver como el acceso de mujeres a la educación se ha incrementado notablemente en las últimas décadas.

De esta manera, podríamos afirmar la existencia de una igualdad de género en los centros escolares. Pero no debemos olvidar que esto se ha conseguido en un periodo relativamente pequeño de tiempo. Muchas de nuestras abuelas no sabían o saben leer o escribir. El recuerdo de una sociedad gobernada únicamente por hombres está aun latente en nuestra cultura, y sigue existiendo desgraciadamente. Es por ello que debemos seguir trabajando para que las nuevas generaciones rompan finalmente con esta discriminación de género.

La llegada de un colectivo inmigrante a los centros españoles en las últimas décadas hace necesaria el refuerzo de información en cuanto a igualdad de género. En el curso 2014/2015 3 los principales países de origen de inmigrantes matriculados en centros españoles en primaria y en educación secundaria obligatoria eran: de origen africano (Marruecos y Argelia); de procedencia europea (Rumanía); de América del Sur (Bolivia,

3 Último curso con datos detallados facilitado por el Ministerio de Educación, Cultura y Deporte. 
Colombia, Ecuador y Perú, entre los más numerosos) y de origen asiático (China y Pakistán, entre otros)

\section{HOMBRES}

\begin{tabular}{|c|c|c|c|c|c|}
\hline \multirow[b]{2}{*}{ Continente } & \multirow[b]{2}{*}{ Primaria } & \multirow[b]{2}{*}{ ESO } & ; Principales & \multirow[b]{2}{*}{ Primaria } & \multirow[b]{2}{*}{ ESO } \\
\hline & & & $\begin{array}{l}\text { I países de } \\
\text { origen }\end{array}$ & & \\
\hline \multirow{2}{*}{ África } & \multirow{2}{*}{46.396} & \multirow{2}{*}{19.832} & Irgelia & 2.009 & 863 \\
\hline & & & Marruecos & 37.202 & 15.888 \\
\hline Europa (UE) & 38.276 & 25.659 & I Rumanía & 19.917 & 12.493 \\
\hline \multirow{3}{*}{ América (Sur) } & \multirow{3}{*}{18.537} & \multirow{3}{*}{28.460} & I Bolivia & 2.870 & 4.518 \\
\hline & & & Colombia & 3.008 & 4.932 \\
\hline & & & I Ecuador & 4.129 & 7.695 \\
\hline \multirow{2}{*}{ Asia } & \multirow{2}{*}{13.298} & \multirow{2}{*}{8.420} & I China & 7.009 & 4.191 \\
\hline & & & Pakistán & 2.545 & 1.900 \\
\hline
\end{tabular}

\section{MUJERES}

\begin{tabular}{|c|c|c|c|c|c|}
\hline Continente & Primaria & ESO & $\begin{array}{l}\text { Principales } \\
\text { I países de } \\
\text { origen }\end{array}$ & Primaria & ESO \\
\hline \multirow{2}{*}{ África } & \multirow{2}{*}{42.385} & \multirow{2}{*}{17.967} & Argelia & 1.869 & 755 \\
\hline & & & Marruecos & 34.012 & 14.610 \\
\hline Europa (UE) & 36.194 & 25.158 & I Rumanía & 18.670 & 12.296 \\
\hline \multirow{3}{*}{ América (Sur) } & \multirow{3}{*}{17.786} & \multirow{3}{*}{28.256} & I Bolivia & 2.849 & 4.612 \\
\hline & & & I Colombia & 2.744 & 4.655 \\
\hline & & & I Ecuador & 3.871 & 7.707 \\
\hline \multirow{2}{*}{ Asia } & \multirow{2}{*}{12.189} & \multirow{2}{*}{6.808} & I China & 6.974 & 3.801 \\
\hline & & & Pakistán & 1.987 & 1.155 \\
\hline
\end{tabular}

De forma breve, veamos cuál es la situación de la mujer en estos países de fuera de nuestras fronteras, pero antes, es fundamental recordar que todas estas regiones están trabajando por conseguir la igualdad de género en todos los ámbitos. La situación de la mujer en países que forman parte del mundo islámico, como pueden ser Marruecos y Argelia, se encuentra en proceso de transformación. Con una fuerte herencia patriarcal, la sociedad islámica tenía establecido que la mujer perteneciera, únicamente, a la esfera de lo privado, siempre en un segundo plano. Es lógico que esto nos suene; otras sociedades occidentales han tenido, en el pasado, este comportamiento hacia el género femenino, pero cambiaron su forma de pensar mucho antes que otras sociedades. Es lo que está sucediendo en el mundo islámico. Como bien explica Terrón (2012: 243)

Determinadas sociedades árabes, en mayor o menor medida, están progresando tanto a nivel de comportamientos como de mentalidad. Lo cual implica transformaciones profundas que necesitan de tiempo y, desde nuestro punto de vista, de una apuesta importante en la mejora de los sistemas educativos para que pueda garantizarse una educación universal y de calidad sin distinción de género ni de nivel socioeconómico de la familia.

Caracterizados por una educación patriarcal, personas inmigrantes de origen islámico han llegado a nuestras aulas. La mayoría estará de acuerdo que la posición de la mujer y el hombre en la sociedad debe ser la misma, pero existen minorías que todavía defienden la supremacía del hombre. Destaco en este punto la importancia de trabajar en los centros educativos la igualdad de género. En palabras de Terrón (2012:250)

Finalmente, señalar la importancia que la educación está teniendo en este proceso de cambio del que muchas mujeres del mundo árabe son protagonistas, lleven o no velo. Es la clave que propicia el desarrollo de cualquier sociedad, pero exige, necesariamente, una fuerte inversión y compromiso de los gobiernos en ella sin discriminación alguna y con el propósito de perseguir la equidad para eliminar todo tipo de desigualdades sociales.

Pasemos a ver cuál es la situación de la mujer en otro de los países cuyos inmigrantes han incrementado el número de alumnado de las aulas españolas: Rumanía. Al igual que sucede en otras culturas, la población rumana mantiene patrones patriarcales de división de género. Si bien la posición de la mujer en la sociedad rumana está cambiando, muchos individuos de esta nacionalidad - tanto mujeres como hombresconservan la idea de que el hombre debe ser el cabeza de familia. Podemos ver un claro ejemplo en palabras de Suárez y Crespo (2007:248-248)

Estas mujeres, aun habiendo sido cabeza de cadena migratoria familiar, mantiene en sus discursos la idea de que es el varón quien debería haber tomado la iniciativa de emigrar, puesto que a él le corresponde proveer a la familia de seguridad material:

¿Cómo me divorcié de mi marido? Yo creo que ¿sabes? Fue una cosa también ehhh...cómo se llama, ven...venganza.[...] Creo que fue...fue un poco de todo ¿sabes? NO. Pienso yo, algun dia cuando penso mejor, creo que fue así. Pienso asi "tu me dejaste a mi venirme a España, en primer lugar, para estar tú tranquilo que tienes dinero, para estar tu tranquilo que puedes beber cuando te da la gana" porque, de estar en España no creo que bebía tanto, digo yo. Y cuando le llamo por teléfono siempre por aquí, borracho que no podía hablar [RO-11, mujer rumana, 48 años] 
Bolivia, Colombia y Ecuador son países que se encuentran en América del Sur cuya principal característica común es que hablan la lengua española, hecho que ha propiciado la entrada de un gran número de inmigrantes a territorio español ya que no tienen como inconveniente la barrera del idioma. En origen, la sociedad latinoamericana estaba provista de ideas que discriminaban a la mujer. Hoy en día, como en el resto de países, la igualdad entre sexos está ganando terreno, pero aún podemos encontrar en muchas regiones está discriminación de género. Como explica Ruiz y Bonometti (2010:77)

La discriminación de género en esta región viene marcada por cuestiones tales como la religión, unos persistentes valores culturales asentados en ideas machistas y de sumisión de la mujer [...] Junto a ello, el empleo deficitario y precario, el escaso acceso a los recursos económicos, como la posesión de tierras se muestran como aspectos estructurales que impiden avanzar en la reducción de las desigualdades [...]. En América Latina la condición de las mujeres no se puede entender sin considerar su estructura social y la falta de cohesión social en entornos con estructuras de gobierno que, en líneas generales, podemos caracterizar como débiles. A pesar de ello, se observan importantes avances especialmente desde la Conferencia de Beijing de 1995 que es el momento crucial a partir del cual se impulsan acciones promovidas desde colectivos de mujeres.

Finalmente, en países asiáticos, como China, la situación de la mujer en la sociedad ha cambiado mucho en los últimos años. Su perspectiva de género es diferente a la que encontramos en otros países que hemos visto como Rumania o Ecuador. Veamos como lo explica Sáiz (2009:186-187)

El género, al igual que otros términos como derechos humanos, pobreza, desarrollo, medio ambiente, etc., aplicados a la situación de las mujeres en China, supone la internacionalización de una agenda gubernamental que formalmente homogeniza a los países en el uso de un lenguaje común. Pero lo hace desde el lado de las mujeres, es decir, tomando a los hombres como el referente de igualdad, los sujetos sociales de cualidad en la sociedad china. La desigualdad entre hombres y mujeres, por tanto, radica en la excelencia y en la medida en que las mujeres chinas son las que ocupan el lado de la desigualdad, la conclusión es que ellas tienen menor cualidad que sus compatriotas varones, olvidando que las razones de la desigualdad entre los sexos se da en la estructura social basada en el poder masculino, no en su cualidad.

La entrada de este colectivo inmigrante puede traer consigo una información errónea sobre la igualdad entre hombres y mujeres. Aunque en sus respectivos países de origen se está trabajando en conseguir erradicar la discriminación de género en todos los ámbitos, en algunos de estos países el inicio de esta lucha es más reciente, por lo que algunos individuos inmigrantes integrados en la sociedad española pueden mantener una estructura patriarcal en sus familias cuyos hijos se incorporan a los centros educativos. En este tipo de estudiantes, cambiarles de forma de pensar en algo que han vivido y viven dentro de su núcleo familiar, es difícil. Pueden creer que solo se trata de una idea distinta defendida por ciertos individuos que habitan su nuevo entorno. No es así. Debemos enseñarles que la igualdad de género es algo global, que no se da únicamente en una cierta comunidad. La información facilitada a través de los medios de comunicación, además de las medidas que toman en centros educativos, son de gran ayuda para que en un aula caracterizada por su diversidad cultural, no exista diferenciación de género por parte de los estudiantes. Por otro lado, herramientas tecnológicas que puedan ellos manejar, como por ejemplo, internet o cualquier dispositivo multimedia, pueden servir como vehículo para trabajar la materia de igualdad de manera más individualizada.

\section{El USO DE LAS TIC EN EL AULA DE IDIOMAS COMO HERRAMIENTAS DE} INFORMACIÓN SOBRE IGUALDAD DE GÉNERO

La versatilidad que ofrece la enseñanza de idiomas a la hora de utilizar diferentes herramientas -en nuestro caso tecnológicas- es de gran utilidad para introducir en el aula multicultural conceptos básicos sobre igualdad, tolerancia y respeto, no sólo hacia estudiantes de otras nacionalidades, sino también, entre los alumnos y alumnas del centro. El uso de las nuevas tecnologías es una realidad en los centros educativos de hoy en día. Desde hace años, ya se utilizan las pizarras digitales, todos los centros están provistos de un aula ALTHIA para el manejo de programas informáticos o internet, y gran cantidad de profesorado innova en sus clases con el uso de diferentes aplicaciones que las nuevas tecnologías ofrecen. El uso del e-learning, o aprendizaje electrónico, se ha extendido a todos los ámbitos formativos. Según Cabrero (2006: 3) las principales ventajas que ofrece este tipo de enseñanza son

- Pone a disposición de los alumnos un amplio volumen de información.

- Facilita la actualización de la información y de los contenidos.

- Flexibiliza la información, independientemente del espacio y el tiempo en el cual se encuentren el profesor y el estudiante.

- Permite la deslocalización del conocimiento.

- Facilita la autonomía del estudiante.

- Propicia una formación just in time y just for me.

- Ofrece diferentes herramientas de comunicación sincrónica y asincrónica paran los estudiantes y para los profesores.

- Favorece una formación multimedia. 
- Facilita una formación grupal y colaborativa.

- Favorece la interactividad en diferentes ámbitos: con la información, con el profesor y entre los alumnos.

- Facilita el uso de los materiales, los objetos de aprendizaje, en diferentes cursos.

- Permite que en los servidores pueda quedar registrada la actividad realizada por los estudiantes.

- Ahorra costos y desplazamiento.

Como podemos ver, las ventajas que ofrece este tipo de enseñanza son varias. Entre las que quiero destacar, está la posibilidad de iniciar el proceso en cualquier lugar y en cualquier hora, característica importante ya que, aunque estemos hablando del aprendizaje de idiomas dentro de un aula, estos estudiantes, gracias a las nuevas tecnologías, pueden continuar fuera del centro ofreciendo la posibilidad de reforzar los contenidos aprendidos en clase. Cognitivamente hablando, podemos introducir diferentes conceptos relacionados con los principios de igualdad, tolerancia y respeto hacia otras culturas, eliminando la discriminación de género, a través de las distintas actividades que realiza el estudiante por medio de recursos diseñados por aplicaciones tecnológicas. Las TIC se han convertido, por lo tanto, en un medio de aprendizaje que resulta atractivo y diferente a la hora de aprender y debemos aprovecharnos de esta cualidad.

Ahora bien, el uso de herramientas o aplicaciones tecnológicas en el aula no trata simplemente de coger una de estas herramientas o aplicaciones y hacer uso de ella. Para que se produzca un correcto proceso de enseñanza-aprendizaje, en necesario que el docente establezca unos objetivos de aprendizaje y determinar qué proceso ha de seguir el estudiante para cumplir esos objetivos. En este artículo, examinaremos cómo la conocida Taxonomía de Bloom es válida para obtener resultados positivos en la enseñanza de lenguas incluyendo, a su vez, los principios de igualdad de género que queremos incorporar en el aula.

\section{TAXONOMÍA DE BLOOM Y TIC EN EL AULA DE IDIOMAS}

En 1956, Benjamín Bloom publicó su taxonomía original. Desde entonces, se han editado varias revisiones, teniendo en cuenta los cambios y desarrollo educativos que se han ido produciendo. Esta taxonomía nació a partir de la tarea de clasificar objetivos educativos. Originalmente, Bloom junto con otros investigadores desarrollaron un sistema de clasificación teniendo en cuenta tres aspectos: el cognitivo, el afectivo y el psicomotor. Principalmente, esta taxonomía tiene una estructura jerárquica que va des de lo más simple a lo más complejo, hasta conseguir llegar a la evaluación. Son seis: conocimiento; comprensión; aplicación; análisis; síntesis; y evaluación. Estos niveles en que queda dividida la taxonomía se alcanzan a través de diferentes actividades que nuestros alumnos y alumnas deben superar, hasta conseguir el objetivo final que es marcado por el docente.

Una de sus actualizaciones fue diseñada por Andrew Churches en 2008. Ofrece una actualización de la Taxonomía de Bloom para la era digital. Veamos un ejemplo de ésta aplicada al aprendizaje de idiomas sin olvidar los mismos principios de igualdad, tolerancia y respeto que tantas veces hemos repetido a lo largo de esta exposición.

Churches (2008) $)^{4}$ establece seis niveles:

- Recordar, que significa recuperar, rememorar o reconocer conocimientos que están en la memoria.

- Comprender, que es construir significado a partir de diferentes tipos de funciones, sean estas escritas o gráficas.

- Aplicar, o llevar a cabo un procedimiento durante el desarrollo de una representación.

- Analizar, es decir, descomponer en partes materiales o conceptuales y determinar cómo estas se relacionan o se interrelacionar entre sí con una estructura completa, o con un propósito determinado.

- Evaluar o hacer juicios en base a criterios y estándares utilizando la comprobación y la crítica.

- Crear, es decir, juntar los elementos para formar un todo coherente y funcional; generar, planear o producir para reorganizar elementos en un nuevo patrón o estructura.

¿Qué actividades pueden desarrollarse a través de las TIC en el aula de idiomas aplicando esta taxonomía? Pues son muy numerosas $\mathrm{y}$, constantemente, van apareciendo nuevas gracias a la gran velocidad que las nuevas tecnologías evolucionan y se expanden. Veamos algunos ejemplos ${ }^{5}$ :

$4 \quad$ En http://www.eduteka.org/TaxonomiaBloomjCuadro.php3

5 Esta tabla muestra algunos ejemplos de actividades digitales que pueden aplicarse en el aula de idiomas por medio de diferentes herramientas o aplicaciones. Son únicamente algunos ejemplos, Churches (2008) explica muchos más en su wiki http://edorigami.wikispaces.com 


\begin{tabular}{|c|c|c|}
\hline & Actividades digitales & $\begin{array}{l}\text { Medio digital que puede aplicarse en el aula } \\
\text { de idiomas }\end{array}$ \\
\hline Recordar & $\begin{array}{l}\text { 1. Narrar o relatar } \\
\text { 2. Examen o prueba } \\
\text { 3. Flashcards } \\
\text { 4. Buscadores básicos }\end{array}$ & $\begin{array}{l}\text { 1.Procesador de texto, mapas mentales, } \\
\text { herramientas de presentación } \\
\text { 2. Procesador de texto, herramientas en línea } \\
\text { 3. Moodle, Hot Potatoes } \\
\text { 4.Motores de búsqueda, catálogo de bibliotecas, } \\
\text { clearinghouses }\end{array}$ \\
\hline Comprender & $\begin{array}{l}\text { 1. Resumir } \\
\text { 2. Explicar } \\
\text { 3. Alimentar un diario } \\
\text { en blog } \\
\text { 1. Publicar a diario } \\
\text { 2. Etiquetar, registrar } \\
\text { comentarios }\end{array}$ & $\begin{array}{l}\text { 1. Procesador de texto, mapas conceptuales, } \\
\text { diarios en blogs. } \\
\text { 2. Presentaciones multimedia, herramientas de } \\
\text { audio y video, mapas mentales. } \\
\text { 1. Bloglines, blogger, Wordpress } \\
\text { 2. Blogging, Myspaces, Facebook, Twitter, } \\
\text { Blogger } \\
\text { 3. Foros de discusión, lectores de archivo (PDF) }\end{array}$ \\
\hline Aplicar & $\begin{array}{l}\text { 1. Ilustrar } \\
\text { 2. Demostrar } \\
\text { 3. Presentar } \\
\text { 4. Ejecutar } \\
\text { 5. Editar }\end{array}$ & $\begin{array}{l}\text { 1. Corel, GIMP, Paint, herramientas en línea } \\
\text { 2. Presentaciones gráficas, conferencias usando } \\
\text { audio o video } \\
\text { 3. Autoublicaciones, Presentador Multimedia, } \\
\text { Google Docs, Skype. } \\
\text { 4. Podcast, vodcast, películas, grabación de } \\
\text { audio. } \\
\text { 5. Herramientas de sonido y video, editar un } \\
\text { Wiki, autopublicaciones. }\end{array}$ \\
\hline Analizar & $\begin{array}{l}\text { 1. Usar bases de datos } \\
\text { 2. Resumir } \\
\text { 3. Elaborar mapas que } \\
\text { establecen relaciones } \\
\text { 4. Informar } \\
\text { 5. Graficar }\end{array}$ & $\begin{array}{l}\text { 1. bases de datos que usan MySQL y Microsoft } \\
\text { Access, Wikis, sistemas de información } \\
\text { geográfica. } \\
\text { 2. Procesador de texto, publicar en Web } \\
\text { 3. Mapas conceptuales, Cmap Tools } \\
\text { 4. Procesador de texto, Deskop Publishing, } \\
\text { Hoja de cálculo } \\
\text { 5. Digitalizadores, herramientas en línea }\end{array}$ \\
\hline
\end{tabular}

Revista Internacional de Culturas y Literaturas, abril 2017

\begin{tabular}{l|l|l} 
& & $\begin{array}{l}\text { 1. Procesador de texto, podcasting, mapas } \\
\text { conceptuales, mensajería instantánea, correo, } \\
\text { conferencias por video. }\end{array}$ \\
Evaluar & $\begin{array}{l}\text { 2. Procesador de texto, blogs, wikis, páginas } \\
\text { web, mapas mentales }\end{array}$ \\
2. Evaluar & 3. Opinar & 3. Procesador de texto \\
4. Comentar, moderar & 4. Paneles de discusión, foros, blogs, wikis, \\
5. Trabajar en redes & $\begin{array}{l}\text { Twitter, salas de conversación } \\
\text { 5. Conferencias en audio y video, correo } \\
\text { electrónico, telecomunicaciones, mensajería } \\
\text { instantánea, clases virtuales }\end{array}$ \\
\hline 1. Producir películas & 1. Movie Maker, Pinnacle, Premier, Animoto \\
2. Presentar & 2. Powerpoint, Zoho, Comic life \\
3. Narrar historias & 3. Procesador de texto, Mixbooks, photostory \\
4. Programar & 5. Scratch, Alice, Game Maker. \\
5. Planear & 5. Inspiration, Cpmap tolos, Free mind
\end{tabular}

\section{CONCLUSIONES}

Como hemos visto a través de estas páginas, conseguir la igualdad de género es una tarea que hoy en día, en pleno siglo XXI, se ha de seguir trabajando. Es cierto que, en el ámbito educativo, se trabajan valores sobre la no discriminación, de tolerancia y respeto hacia cualquier individuo, pero, aún así, todavía existen núcleos en los que la jerarquía patriarcal existe, otorgando a la mujer un papel secundario en la sociedad. Por otro lado, debido a la llegada de un colectivo inmigrante bastante numeroso desde la década de 1990, cuyas culturas pueden haber evolucionado más lentamente en cuanto a la igualdad de género, puede provocar la necesidad de reforzar la información en cuanto a este tema debido a que, muchos de estos estudiantes, viven en núcleos familiares cuya jerarquía patriarcal es aún latente.

La actual ley de educación vigente (LOMCE) al igual que su predecesora (LOE) incluyen elementos que permiten la prevención de la violencia de género, informando sobre la promoción, desde la infancia, de la igualdad efectiva entre hombres y mujeres. En su artículo 1, la LOMCE, informa de la importancia de conseguir ${ }^{6}$

$$
\begin{aligned}
& \text { La equidad, que garantice la igualdad de oportunidades para el pleno desarrollo de la } \\
& \text { personalidad a través de la educación, la inclusión educativa, la igualdad de derechos y } \\
& \text { oportunidades que ayuden a superar cualquier discriminación y la accesibilidad universal } \\
& \text { a la educación, y que actúe como elemento compensador de las desigualdades personales, } \\
& \text { culturales, económicas y sociales, con especial atención a las que se deriven de cualquier } \\
& \text { tipo de discapacidad. }
\end{aligned}
$$

6 Ley Orgánica 8/2013, de 9 de diciembre, para la mejora de la calidad educativa 
Y es así como, en los centros educativos españoles, no se debe frenar la educación en materia de igualdad.

Las tecnologías de la información y la comunicación pueden servir como herramienta para lograr introducir estos principios en nuestros estudiantes. Se ha elegido la materia de enseñanza de idiomas debido a que el uso de las TIC son de gran ayuda para el aprendizaje de lenguas. Otro factor que caracteriza al aprendizaje de lenguas es la existencia de una mayor cantidad de mujeres que inician estudios de idiomas, cuya cifra duplica al número de hombres que deciden estudiar esta materia ${ }^{7}$. La necesidad de crear entornos reales para un mejor aprendizaje de una L2, facilita la introducción de estos valores que son aprendidos cognitivamente por el estudiante gracias al uso de aplicaciones tecnológicas.

Pero para un buen aprendizaje, es necesario establecer unos objetivos que el alumno o alumna deben alcanzar. Por este motivo, la taxonomía de Bloom, modificada para la era digital, resulta de gran ayuda. A través de sus distintos niveles y, por medio de actividades digitales que pueden realizarse a través de multitud de aplicaciones al alcance de todos los estudiantes, tanto mujeres como hombres, es posible conseguir, de forma efectiva, los objetivos de aprendizaje además de trabajar los valores de igualdad, tolerancia y respeto hacia personas de distinto sexo o culturas.

En resumen, la variable de género en la enseñanza de idiomas es un factor a tener en cuenta a la hora de trabajar con alumnos y alumnas de cualquier ciclo de enseñanza, sobre todo, si se tratan de aulas multiculturales cuyos estudiantes de diferentes orígenes no han terminado de aceptar, debido a la falta de información o de educarse en ambientes con ideas patriarcales, la importancia que adquiere, en nuestra sociedad la igualdad de género. Gracias a las tecnologías de la información y la comunicación puede acelerarse el proceso de alcanzar una plena heterogeneidad entre todas las culturas.

\section{REFERENCIAS BibLIOGRÁFICAS}

Amo, M. C., "La educación de las mujeres en España: de la "amiga" a la Universidad", CEE Participación educativa, 11, julio 2009, p. 9.

Cabrero J., "Bases pedagógicas del e-learning" Revista de Universidad y Sociedad del Conocimiento, Vol. 3, nº 1, abril 2006, pp. 3-4. Internet. 30-08-2016. <http://www.uoc edu/rusc/3/1/dt/esp/cabero.pdf>

Churches A., “Taxonomía de Bloom para la Era Digital”. 2008. Internet. 19-09-2016. < http://edorigami. wikispaces. com>.

7 Según datos estadísticos del Ministerio de Educación, Cultura y Deporte, en el curso 2014/2015, el alumnado matriculado en enseñanza de idiomas asciende a un total de 149.280 hombres y 299.186 mujeres.
Flecha C., “Desequilibrios de género en educación en la España Contemporánea: causas, indicadores y consecuencias", Revista Internacional de Ciencias Sociales, n⿳⺈ 33 , 2014, p.52.

Godeau D., Rinken S., Martínez de Lizarrondo A., Moreno G., La integración de los inmigrantes en España: una propuesta de mediación a escala regional, Documentos del Observatorio Permanente de la Inmigración. 2014. Internet. 02-08-2016. < http:// extranjeros. empleo.gob.es/es/ObservatorioPermanenteInmigracion/ Publicaciones /fichas/archivos/OPI_30.pdf>.

Lomce, Ley Orgánica de mejora de la calidad educativa. Internet. 28-09-2016. < [https:// www.boe.es/diario_boe/txt.php?id=BOE-A-2013-12886>

Mnisterio de Educación, Cultura y Deporte, Las cifras de la educación en España. Curso 2013/2014. Edición 2016, p. 8. Internet. 24-08-2016. < http://www.mecd.gob.es/ servicios-al-ciudadano-mecd/estadisticas/educacion/indicadores-publicacionessintesis/cifras-educacion-espana/2013-14.html>

Ruiz, S., Bonometti, P., “Las mujeres en América latina: indicadores y datos”. Revista Ciencias Sociales 126-127:75-87/2009-2010 (IV-I), 2010, p.77, Internet. 07-09-2016. < http:// unpan1.un.org/intradoc/groups/public/documents/icap/unpan046955.pdf>

Saiz, A., “Mujeres y género en la sociedad china contemporánea”, J. Julià-Muné, (ed.), Visions de la Xina: cultura multimil-lenària, Lleida, Institut d'Estudis Ilerdencs de la Diputació de Lleida, 2009, pp. 169-190.

Sales, A., García R., Programas de educación intercultural, Desclée de Brouwer, Bilbao, 1997, pp. 11-19.

Song, S., "Multiculturalism", E. N. Zalta (ed.), The Stanford Encyclopedia of Philosophy (Spring 2014 Edition), 2010. Internet. 02-08-2016. < http://plato.stanford. edu/archives/spr2014/ entries/multiculturalism/>

Suárez, L., Crespo, P., “Familias en movimiento. El caso de las mujeres rumanas en España", Migracione,s 21 2007, pp. 247-248.

Terrón, T., "La mujer en el Islam. Análisis desde una perspectiva socioeducativa”. Revista El Futuro del Pasado, no 3, 2012, pp. 237-254. Internet. 05-09-2016. < [https:// dialnet.unirioja.es/descarga/articulo/3941216.pdf>

Unesco, educación en la escuela. Internet. 16-08-2016. < http://www.unesco.org/new/ es/education/themes/leading-the-international-agenda/education-for-sustainabledevelopment/gender-equality/> 